\title{
TRIBUNA DE ECONOMÍA
}

Todos los artículos publicados en esta sección son sometidos a un riguroso proceso de evaluación anónima. 



\title{
Políticas de promoción de las energías eólicas y solar: los casos de Alemania y España*
}

\author{
María Teresa García-Álvarez \\ Rosa María Mariz-Pérez \\ Fernando de Llano Paz \\ Universidad de La Coruña
}

\section{Resumen}

En los últimos años estamos asistiendo a un proceso de desarrollo de las energías renovables en la Unión Europea con la finalidad de contribuir a la reducción de emisiones de gases de efecto invernadero, además de reducir la dependencia energética. No obstante, la normativa europea fija diferentes mecanismos de promoción de estas tecnologías de producción donde los Estados Miembros tienen libertad para utilizar el que mejor se adapte a sus necesidades.

En este trabajo se analizan las características regulatorias así como los resultados alcanzados en energía eólica y en energía solar fotovoltaica y térmica en los países de la Unión Europea con mayor capacidad instalada y producción en tales tecnologías de producción. Los resultados del trabajo ponen de manifiesto el éxito que conlleva el sistema de primas como instrumento de desarrollo de la energía eólica y solar.

Palabras clave: sector eléctrico, energías renovables, regulación.

Clasificación JEL: Q42, Q50, Q58.

\begin{abstract}
In the last years, a process of development of renewable energies is taking place in EU with the aim of reducing greenhouse gas emissions and energy dependency. However, the European normative establishes different promotion mechanisms of renewable energies and Member States have freedom to choose the most adequate mechanism adapted to its needs.

In this paper, we analyze the regulatory characteristics and the results of wind energy and photovoltaic and thermal solar energy in the EU Member States with higher installed capacity and production. The results of the paper show the success that entails the feed-in system as a mechanism to promote the development of such renewable energies.
\end{abstract}

Keywords: electricity industry, renewable energies, regulation.

JEL classification: Q42, Q50, Q58.

* Este trabajo se enmarca dentro de un estudio más amplio desarrollado en el proyecto de investigación de I+D+i «Análisis de los efectos del desarrollo de las energías renovables en la Unión Europea sobre el empleo», concedido por la Xunta de Galicia, con código 10SEC100020PR.

Fecha de conclusión del trabajo: octubre de 2012. 


\section{Introducción}

La promoción de energías renovables es un elemento clave en la Unión Europea, tanto por razones medioambientales como económicas. Así, este tipo de energía contribuye a conseguir los objetivos establecidos por el Protocolo de Kioto. Además, permite la obtención de diversas ventajas socio-económicas, tales como la diversificación de la oferta de energía, la mejora de oportunidades de desarrollo regional y local, la creación de una industria doméstica y la creación de empleo (Moreno y López, 2008).

En este contexto, la participación de los gobiernos es necesaria en la fase inicial de implantación de tales tecnologías de producción para asegurar su desarrollo así como para protegerlas de la competencia directa de las tecnologías convencionales. Este apoyo gubernamental puede ser justificado por dos motivos: corregir las externalidades negativas resultantes del uso de combustibles fósiles y estimular el cambio técnico (Menanteau et al., 2003):

- Corregir las externalidades negativas resultantes del uso de combustibles fósiles. La principal ventaja de las energías renovables, con respecto a la generación de energía convencional, es que contribuyen a la conservación de los bienes públicos (concretamente al aire limpio y a la estabilidad climática). No obstante, los precios de la electricidad no reflejan en su totalidad el coste real de su generación puesto que una parte de los costes ambientales y sociales que llevan asociados siguen siendo externalizados. De ahí, la necesidad de establecer mecanismos que, de alguna manera, corrijan dicha situación.

- Estimular el cambio técnico. Las energías renovables tienen que competir con otras tecnologías de producción establecidas en el sistema, permaneciendo en una posición desfavorable. Así, cuando tales tecnologías entran en el mercado no han alcanzado su rendimiento óptimo en términos de coste y fiabilidad sino que el mismo será conseguido gradualmente como resultado del proceso de aprendizaje con su puesta en marcha o learning by doing (Dosi, 1988). De ahí la necesidad del establecimiento de un sistema de incentivos que permita solventar tales cuestiones.

Debido a tales características, se pone de manifiesto la necesidad del desarrollo de instrumentos que permitan promover las energías renovables. En este contexto, el objetivo del presente trabajo es determinar los factores de éxito (de tales mecanismos de promoción de energía renovable) que han permitido que Alemania y España se sitúen como los dos primeros países de la UE en términos de potencia instalada y capacidad de producción en energía eólica y en energía solar fotovoltaica y térmica.

Para ello, el trabajo se estructura de la siguiente forma: en primer lugar, se estudian las principales características de los distintos mecanismos de promoción de energía renovable utilizados en la UE. A continuación, se analizan las políticas de promoción de energía eólica y solar en Alemania y España. Posteriormente, se rea- 
liza una comparación de tales políticas y se muestran los resultados derivados de su aplicación en ambos países. Finalmente, a partir del análisis realizado, se extraen las principales conclusiones del trabajo que pueden servir como base para el aprendizaje de otros países con respecto a las principales ventajas que conlleva el uso de un sistema de primas.

\section{El desarrollo de las energías renovables en la Unión Europea}

La Directiva 2009/77/CE concede libertad a cada Estado Miembro para elegir el mecanismo de apoyo a las energías renovables que mejor se adecue a sus características. Una revisión de tales medidas aplicadas en la UE pone de manifiesto el establecimiento de tres tipos de mecanismos: $a$ ) el sistema de primas, $b$ ) las subastas competitivas y $c$ ) las cuotas de certificados verdes negociables (ver Cuadro 1 ).

Tal y como hemos comentado, los países de la UE han implantado distintos mecanismos de apoyo $^{1}$ a las tecnologías de producción renovable, con lo que no existe un consenso acerca de cuál es el más adecuado (Vera, 2012). No obstante, la experiencia pone de manifiesto que el desarrollo de un sistema de primas, que permita garantizar una atractiva rentabilidad de las instalaciones renovables, es efectivo en la promoción de la expansión de las energías renovables.

Estos son los casos de Alemania y España, que implantaron un sistema de primas desde las primeras etapas de promoción de energía renovable, y que les ha permitido consolidarse como las dos primeras potencias europeas ${ }^{2}$ en capacidad instalada y producción de este tipo de energía eólica y solar.

${ }^{1}$ Asimismo, tales mecanismos pueden ser complementados con medidas alternativas para promocionar las energías renovables, tales como los incentivos fiscales (VARELA y GARCÍA, 2010), subvenciones a la inversión o subvenciones para la cobertura de gastos corrientes.

${ }^{2}$ No obstante, aunque Alemania y España son las dos primeras potencias europeas en capacidad instalada y producción de electricidad a partir de energías renovables, otros Estados Miembros tienen el liderazgo en términos de porcentaje de energías renovables en el consumo eléctrico total. Este concepto alcanza un valor superior incluso al 50 por 100 en los casos de Austria (60,6 por 100), Suecia (54,7 por $100)$ y Portugal $(50,9$ por 100$)$. 


\section{CUADRO 1 \\ CARACTERÍSTICAS DE LOS SISTEMAS DE PROMOCIÓN DE ENERGÍAS RENOVABLES UTILIZADOS EN EUROPA}

\begin{tabular}{|c|c|}
\hline $\begin{array}{l}\text { Sistemas de apoyo a } \\
\text { las energías renovables } \\
\text { en la Unión Europea }\end{array}$ & Características \\
\hline Sistemas de primas & $\begin{array}{l}\text { Mediante este mecanismo, los productores de energía renovable tienen } \\
\text { derecho a vender toda su producción a la red eléctrica y obtener, por esto, } \\
\text { una retribución basada en un precio fijo o en el precio horario del mercado } \\
\text { eléctrico general más un incentivo que compense el valor ambiental de la } \\
\text { producción renovable. Así, este sistema establece legalmente los precios } \\
\text { o incentivos de cada tecnología de producción renovable (eólica, solar, } \\
\text { biomasa, minihidráulica, etcétera). Esta retribución se establece para } \\
\text { un periodo de tiempo específico que oscila, por lo general, entre diez y } \\
\text { veinte años desde la puesta en marcha de la instalación. } \\
\text { Para un correcto funcionamiento de este mecanismo se requiere que } \\
\text { el incentivo sea lo suficientemente elevado como para garantizar una } \\
\text { rentabilidad atractiva. Cuando se cumple esta condición, los sistemas de } \\
\text { primas han demostrado ser los más efectivos para promover la expansión } \\
\text { de la energía renovable (Fages y Bustos, 2003). }\end{array}$ \\
\hline $\begin{array}{l}\text { Sistema de subastas } \\
\text { competitivas }\end{array}$ & $\begin{array}{l}\text { En este caso, el regulador reserva una proporción de mercado a la } \\
\text { producción mediante energía renovable y se desarrolla la competencia } \\
\text { entre los generadores que utilizan estos recursos. Los distribuidores están } \\
\text { obligados a adquirir el total de la cantidad producida en dicho mercado } \\
\text { reservado. Por tanto, mediante este mecanismo, la competencia se centra } \\
\text { en el precio debido a que las ofertas de producción son clasificadas en } \\
\text { orden creciente de precios hasta que se alcance la cantidad propuesta. } \\
\text { Este instrumento ha sido utilizado principalmente en Reino Unido y } \\
\text { Francia (en este último caso, hasta el año 2000). }\end{array}$ \\
\hline $\begin{array}{l}\text { Cuota de certificados } \\
\text { verdes negociables }\end{array}$ & $\begin{array}{l}\text { El objetivo de este sistema es que la energía producida a partir de fuentes } \\
\text { renovables pueda llegar a convertirse en parte integral del mercado } \\
\text { eléctrico. Para ello, el Estado impone la obligación, a los distribuidores } \\
\text { de electricidad, de que un determinado porcentaje de su suministro, } \\
\text { generalmente creciente en el tiempo, provenga de energía renovable } \\
\text { (cuota fija de electricidad). Al final de cada periodo -normalmente a } \\
\text { nivel anual-, los distribuidores tienen que demostrar su cumplimiento } \\
\text { de la cuota mediante la entrega virtual, a la correspondiente Autoridad } \\
\text { Regulatoria Nacional, de una cantidad de certificados verdes equivalentes } \\
\text { a una cuota de MWh renovables que suministrar (un certificado verde } \\
\text { suele equivaler a un MWh renovable). } \\
\text { Este sistema está siendo utilizado, o próximamente será usado, en } \\
\text { diversos países sobre una base más o menos experimental (Países Bajos, } \\
\text { Dinamarca, Suecia, Italia y Reino Unido). }\end{array}$ \\
\hline
\end{tabular}

FUENTE: Elaboración propia. 


\section{La política de promoción de energías renovables basada en el sistema de primas: análisis de los casos de Alemania y España}

El éxito en la promoción de energías renovables ${ }^{3}$ en los países analizados en este trabajo viene dado, en buena medida, en el marco regulatorio utilizado que se ha basado en un sistema de primas. A continuación, se realizará un análisis pormenorizado de las características regulatorias de los sistemas de primas de Alemania y España para determinar sus factores de éxito (ver Cuadro 2 y Cuadro 5).

\section{CUADRO 2}

\section{RESUMEN DE LAS PRINCIPALES REGULACIONES EN ENERGÍA RENOVABLE EN ALEMANIA}

\begin{tabular}{|l|l|}
\hline $\begin{array}{l}\text { Regulación de las energías } \\
\text { renovables }\end{array}$ & \multicolumn{1}{c|}{ Características } \\
\hline $\begin{array}{l}\text { Regulación en el año 1974 } \\
\text { (después de la primera cri- } \\
\text { sis del petróleo) }\end{array}$ & $\begin{array}{l}\text { Esta política consistió casi exclusivamente en la promoción de la in- } \\
\text { vestigación (desde la preparación de los trabajadores hasta el desarro- } \\
\text { llo de prototipos y producción en laboratorio). }\end{array}$ \\
\hline $\begin{array}{l}\text { Ley de Competencia Na- } \\
\text { cional (1979) }\end{array}$ & $\begin{array}{l}\text { Esta ley conlleva la realización de los primeros esfuerzos en promover } \\
\text { la demanda de energías renovables mediante el uso de la tarifa. Así, el } \\
\text { gobierno obliga a los distribuidores a comprar electricidad producida a } \\
\text { partir de fuentes de energía renovable en su área de oferta basada en el } \\
\text { principio de los costes evitables. }\end{array}$ \\
\hline $\begin{array}{l}\text { Sistema regulatorio de apo- } \\
\text { yo a la tarifa (1991-2000) }\end{array}$ & $\begin{array}{l}\text { Conlleva el establecimiento de varias medidas para la creación de mer- } \\
\text { cados de tecnología de producción renovable. Entre los mismos, se } \\
\text { encontraban el «Programa Eólico con 100/250 MW», el «Programa de } \\
\text { 1.000 Tejados Solares» y el establecimiento de un sistema de primas } \\
\text { como medida de promoción de determinadas tecnologías de produc- } \\
\text { ción (ver Cuadro 3). Con este marco regulatorio, las empresas eléctri- } \\
\text { cas tienen que conectar a la red a los generadores de energía renovable } \\
\text { y adquirir la electricidad. Su principal objetivo venía dado por «nivelar } \\
\text { el campo de juego» de las energías renovables mediante el desarrollo } \\
\text { de primas que consideraran los costes externos de la generación de } \\
\text { energía convencional. }\end{array}$ \\
\hline
\end{tabular}

FUENTE: Elaboración propia.

\footnotetext{
${ }^{3}$ Aunque a lo largo del presente trabajo se hará referencia a la capacidad instalada y la producción de electricidad a partir de energías renovables, es necesario considerar que este tipo de tecnologías de producción se utiliza no sólo en la producción de electricidad sino también en la producción de calor y refrigeración así como en transporte.
} 


\section{CUADRO 2 (continuación)}

\section{RESUMEN DE LAS PRINCIPALES REGULACIONES EN ENERGÍA RENOVABLE EN ALEMANIA}

\begin{tabular}{|l|l|}
\hline $\begin{array}{c}\text { Regulación de las energías } \\
\text { renovables }\end{array}$ & \multicolumn{1}{c|}{ Características } \\
\hline $\begin{array}{l}\text { Ley de Energías Renova- } \\
\text { bles (EEG) (2000) y sus en- } \\
\text { miendas en los años 2004 y } \\
2009\end{array}$ & $\begin{array}{l}\text { La finalidad de esta regulación es incrementar la proporción de ener- } \\
\text { gías renovables en el suministro eléctrico. En este contexto, los pro- } \\
\text { ductores de energía renovable reciben un pago fijo durante quince o } \\
\text { veinte años por la energía producida y las empresas comercializadoras } \\
\text { están obligadas a darles preferencia en su suministro. La cantidad a } \\
\text { pagar vendrá dada en función del tipo de energía renovable y su ubi- } \\
\text { cación (ver Cuadro 4), importe que se reducirá a medida que transcu- } \\
\text { ran los años. La finalidad que se persigue con este sistema es que las } \\
\text { energías renovables vayan desarrollando su tecnología con el objetivo } \\
\text { de hacerla más competitiva y que cada vez necesiten menos subsidios. }\end{array}$ \\
\hline
\end{tabular}

FUENTE: Elaboración propia.

\section{CUADRO 3}

PRIMAS ESTABLECIDAS EN LA ENERGÍA EÓLICA Y SOLAR CON EL SISTEMA REGULATORIO DE APOYO A LA TARIFA, 1 ENERO 1991-1 ABRIL 2000 (En pfennings*)

\begin{tabular}{|l|c|c|c|c|c|c|c|c|c|c|}
\hline & $\mathbf{1 9 9 1}$ & $\mathbf{1 9 9 2}$ & $\mathbf{1 9 9 3}$ & $\mathbf{1 9 9 4}$ & $\mathbf{1 9 9 5}$ & $\mathbf{1 9 9 6}$ & $\mathbf{1 9 9 7}$ & $\mathbf{1 9 9 8}$ & $\mathbf{1 9 9 9}$ & $\mathbf{2 0 0 0}$ \\
\hline $\begin{array}{l}\text { Energía eólica, solar } \\
\text { (90 por 100 del pre- } \\
\text { cio de venta) }\end{array}$ & 16,61 & 16,53 & 16,57 & 16,93 & 17,28 & 17,21 & 17,5 & 16,79 & 16,52 & 16,13 \\
\hline
\end{tabular}

NOTA: $* 100$ pfennings $=0,51$ euros.

FUENTE: LAUBER y MEZ (2004).

\section{CUADRO 4}

\section{PRIMAS ESTABLECIDAS EN LA ENERGÍA EÓLICA Y SOLAR CON LA LEY DE ENERGÍAS RENOVABLES* (En céntimos de euro/kWh)}

\begin{tabular}{|l|c|c|c|c|c|c|c|c|c|c|c|c|}
\hline & $\mathbf{2 0 0 0}$ & $\mathbf{2 0 0 1}$ & $\mathbf{2 0 0 2}$ & $\mathbf{2 0 0 3}$ & $\mathbf{2 0 0 4}$ & $\mathbf{2 0 0 5}$ & $\mathbf{2 0 0 6}$ & $\mathbf{2 0 0 7}$ & $\mathbf{2 0 0 8}$ & $\mathbf{2 0 0 9}$ & $\mathbf{2 0 1 0}$ & $\mathbf{2 0 1 1}$ \\
\hline Eólica & 9,1 & 9,1 & 9 & 8,8 & 8,7 & 8,6 & 8,4 & 8,3 & 8,2 & 8,1 & 7,9 & 7,9 \\
\hline $\begin{array}{l}\text { Radiación } \\
\text { solar }\end{array}$ & 50,6 & 50,6 & 48,1 & 45,7 & 43,4 & 41,2 & 39,2 & 37,2 & 35,3 & 33,6 & 31,9 & 30,8 \\
\hline
\end{tabular}

NOTA: * El importe de las primas es cambiado, para algunas tecnologías de producción, con las dos enmiendas realizadas a la Ley de Energías Renovables en los años 2004 y 2009. Los principales cambios son comentados en el siguiente apartado (mediante el análisis de las principales tecnologías de producción renovable).

FUENTE: Ley de Energías Renovables. 
En el caso de España, la regulación se ha basado también en un sistema de primas hasta la entrada en vigor del Real Decreto-ley 1/2012 que conlleva la supresión de los incentivos económicos a nuevas instalaciones de producción de energía eléctrica a partir de fuentes renovables. Las características del sistema de primas a las tecnologías de producción renovable en España se recogen en el Cuadro 5.

\section{CUADRO 5}

\section{RESUMEN DE LAS PRINCIPALES REGULACIONES EN ENERGÍA RENOVABLE EN ESPAÑA}

\begin{tabular}{|c|c|}
\hline $\begin{array}{l}\text { Regulación de las } \\
\text { energías renovables }\end{array}$ & Características \\
\hline $\begin{array}{l}\text { Ley } 82 / 1980 \text { de Con- } \\
\text { servación de Energía }\end{array}$ & $\begin{array}{l}\text { Supone el inicio de la regulación en tecnología renovable con el objetivo de } \\
\text { mejorar la eficiencia energética y reducir la dependencia del exterior. }\end{array}$ \\
\hline $\begin{array}{l}\text { Plan Energético Na- } \\
\text { cional 1990-2000 }\end{array}$ & $\begin{array}{l}\text { Establece incentivos para la cogeneración y la producción de energía renovable } \\
\text { mediante el desarrollo de subvenciones a tales tecnologías de producción que } \\
\text { son materializadas en distintas regulaciones, tales como la Orden 28-06-1991, el } \\
\text { R.D. } 2.366 / 1994 \text {, la Orden Ministerial de } 6 \text { de febrero de } 1997 \text {. }\end{array}$ \\
\hline $\begin{array}{l}\text { Ley } 54 / 1997^{*} \text { del } \\
\text { Sector Eléctrico }\end{array}$ & $\begin{array}{l}\text { Supone la liberalización de la industria eléctrica española donde se busca } \\
\text { garantizar el suministro de electricidad a un precio bajo y minimizar el } \\
\text { impacto medioambiental. Para ello, se establece la posibilidad de que los } \\
\text { productores de energía renovable puedan incorporar su energía excedentaria } \\
\text { al sistema a tarifa regulada (vendiendo la energía, de forma directa, a una } \\
\text { empresa distribuidora) o participar directamente en el mercado de producción } \\
\text { de electricidad. Este mecanismo ha permitido un incremento significativo de } \\
\text { inversión en nueva capacidad (Castro-Rodríguez y Siotis, 2010). }\end{array}$ \\
\hline $\begin{array}{l}\text { Real Decreto 2.818/ } \\
1998\end{array}$ & $\begin{array}{l}\text { Esta normativa impulsa el desarrollo de instalaciones de régimen especial } \\
\text { mediante el establecimiento de un sistema de incentivos temporales basado } \\
\text { en primas que permita que su aportación a la demanda energética en España } \\
\text { adquiera una cuota mínima del } 12 \text { por } 100 \text { en el año } 2010 \text {. }\end{array}$ \\
\hline $\begin{array}{l}\text { Los Reales Decretos } \\
436 / 2004 \text { y } 661 / 2007\end{array}$ & $\begin{array}{l}\text { Permiten a los titulares de instalaciones renovables dos posibilidades: a) } \\
\text { vender su excedente de energía eléctrica a un distribuidor por lo que obtendría } \\
\text { una retribución en forma de tarifa regulada y única que se calcula como un } \\
\text { porcentaje de la tarifa eléctrica media o de referencia de cada año o } b \text { ) vender } \\
\text { su excedente de producción de forma directa en el mercado de producción } \\
\text { de electricidad o mediante un contrato bilateral por lo que su remuneración } \\
\text { vendría dada por el precio negociado en el mercado, un incentivo por su } \\
\text { participación y una prima. Mediante esta nueva regulación, la retribución } \\
\text { de cada tecnología de producción no es homogénea sino que viene dada por } \\
\text { el volumen producido y el horizonte temporal de cada planta. Asimismo, } \\
\text { se establece una prima de referencia y unos límites superior e inferior para } \\
\text { la generación de tecnologías de producción renovable que participan en el } \\
\text { mercado (ver Cuadro 6). }\end{array}$ \\
\hline
\end{tabular}

NOTA: * La Ley 17/2007 modifica la Ley 54/1997 con la finalidad de facilitar su adaptación a lo dispuesto en la Directiva 2003/54/CE sobre normas comunes para el mercado interior de electricidad.

FUENTE: Elaboración propia a partir de CIARRETA-ANTUÑANO y GUTIÉRREZ-HITA (2008) y CNE (2011). 


\section{CUADRO 5 (continuación)}

\section{RESUMEN DE LAS PRINCIPALES REGULACIONES EN ENERGÍA RENOVABLE EN ESPAÑA}

\begin{tabular}{|l|l|}
\hline $\begin{array}{l}\text { Regulación de las } \\
\text { energías renovables }\end{array}$ & \multicolumn{1}{c|}{ Características } \\
\hline $\begin{array}{l}\text { Real Decreto 1.578/ } \\
2008\end{array}$ & $\begin{array}{l}\text { Establece un nuevo sistema de tarifas para la energía solar fotovoltaica donde } \\
\text { las nuevas tarifas se basan en la ubicación de este tipo de plantas: las que } \\
\text { están ubicadas en cubiertas (tipo I) y las que están en el suelo (tipo II). }\end{array}$ \\
\hline $\begin{array}{l}\text { Orden ITC/1.723/ } \\
2009\end{array}$ & $\begin{array}{l}\text { Establece una actualización de las tarifas y las primas establecidas para las } \\
\text { tecnologías de producción renovable basadas en cogeneración y residuos. }\end{array}$ \\
\hline $\begin{array}{l}\text { Real Decreto 1.614/ } \\
2010\end{array}$ & $\begin{array}{l}\text { Fija una limitación de las horas equivalentes de funcionamiento de las } \\
\text { instalaciones de producción eólica y solar térmica con derecho a prima, } \\
\text { conllevando una actualización de sus primas. }\end{array}$ \\
\hline $\begin{array}{l}\text { Real Decreto-ley 1/ } 1 / 2012 \\
\text { Establece la suspensión de los procesos de preasignación de retribución } \\
\text { y la supresión de los incentivos económicos para nuevas instalaciones de } \\
\text { producción de energía eléctrica a partir de cogeneración, fuentes de energía } \\
\text { renovables y residuos. Su justificación viene dada porque considera que } \\
\text { España ha cumplido los objetivos establecidos de potencia instalada para la } \\
\text { tecnología eólica y para las tecnologías solar térmica y fotovoltaica. }\end{array}$ \\
\hline
\end{tabular}

FUENTE: Elaboración propia a partir de CIARRETA-ANTUÑANO y GUTIÉRREZ-HITA (2008) y CNE (2011).

Tales incentivos económicos a la promoción de energía renovable eólica y solar fueron revisados por posteriores regulaciones que conllevan una reducción en el importe de dichas primas así como una limitación del número de horas de funcionamiento de tales tecnologías de producción con derecho a prima. En el caso de la energía eólica, el Real Decreto 1.614/2010 establece una reducción de la prima de referencia del 35 por 100 para las instalaciones de energía eólica en tierra y una limitación de las horas equivalentes de funcionamiento de tales instalaciones con derecho a prima.

Limitaciones similares en el número de horas de funcionamiento son aplicadas a las instalaciones solares fotovoltaicas y térmicas (Real Decreto 1.614/2010). Asimismo, el Real Decreto 1.578/2008 conlleva un descenso interanual de las tarifas del 10 por 100 en las instalaciones solares fotovoltaicas. Finalmente, tal y como comentamos previamente, el Real Decreto-ley 1/2012 conlleva una supresión de los incentivos económicos para la promoción de tales tecnologías de producción. 


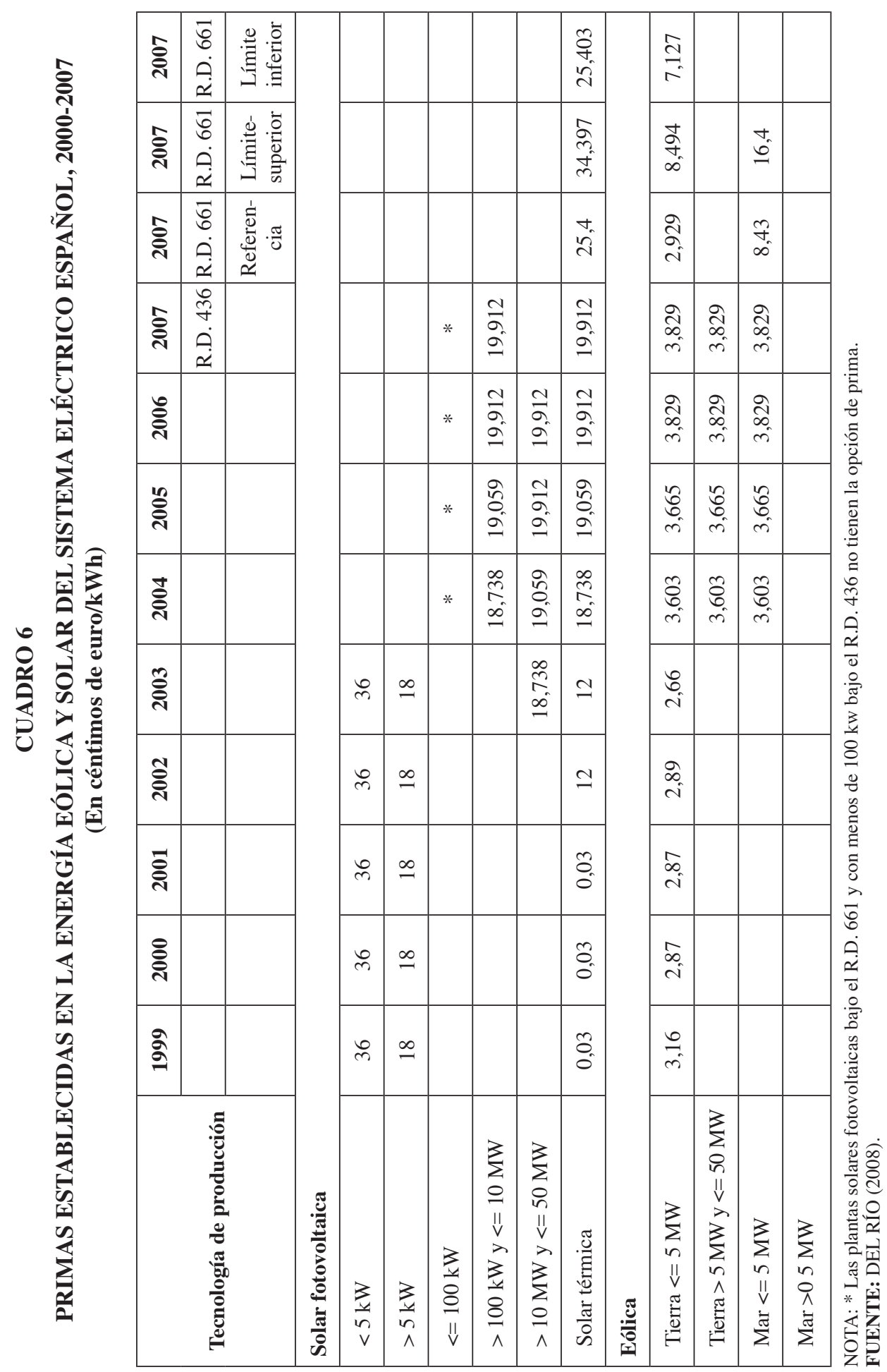




\section{Comparación de los resultados de aplicar un sistema de primas en España y Alemania: características y resultados en la energía eólica y solar}

En los Cuadros 7, 8 y 9 se recogen las principales características de los marcos regulatorios de promoción de las energías renovables aplicados a la energía eólica y a la energía solar térmica y fotovoltaica de Alemania y España.

\section{CUADRO 7 \\ COMPARACIÓN DEL SISTEMA DE PRIMAS DE LA ENERGÍA EÓLICA EN ALEMANIA Y ESPAÑA}

\begin{tabular}{|c|c|}
\hline Alemania & España \\
\hline $\begin{array}{l}\text { Alemania tiene una capacidad instalada de ener- } \\
\text { gía eólica, en el año } 2010 \text {, de } 27.215 \text { MW. Esto } \\
\text { permite un abastecimiento de la demanda del } \\
24,8 \text { por } 100 \text { en dicho estado miembro (Eurob- } \\
\text { server, 2011). } \\
\text { La explicación a este importante desarrollo de } \\
\text { energía eólica en Alemania viene dado de forma } \\
\text { relevante por la legislación aplicada para la pro- } \\
\text { moción de tal tecnología de producción. Destaca } \\
\text { la siguiente normativa: } \\
\text { Sistema de apoyo a la tarifa (1991). Conlleva } \\
\text { una expansión eólica y aprendizaje tecnológico } \\
\text { en el sector (1989: } 20 \text { MW-1995: } 1.100 \text { MW). } \\
\text { Ley de Energías Renovables (2000) (y sus en- } \\
\text { miendas en los años } 2004 \text { y 2009). Suministra } \\
\text { primas atractivas para la energía eólica en tierra } \\
\text { (tarifa inicial: } 8,03 \text { céntimos euro/kWh y, a partir } \\
\text { del año 2008, } 5,07 \text { céntimos euro/kWh) con una } \\
\text { duración entre cinco y veinte años en función del } \\
\text { emplazamiento. También establece primas espe- } \\
\text { ciales para las instalaciones eólicas en alta mar } \\
\text { (tarifa base: } 6,07 \text { céntimos euro/kWh) y repoten- } \\
\text { ciación (repowering)*. } \\
\text { Este marco regulatorio ha sido complementado } \\
\text { con el establecimiento de acuerdos de coopera- } \\
\text { ción entre empresas, institutos de I+D+i y uni- } \\
\text { versidades que han permitido el desarrollo de } \\
\text { avances en tecnología y en la operación de tur- } \\
\text { binas eólicas. Además, el sistema educativo ale- } \\
\text { mán crea especialidades en energía renovable, lo } \\
\text { que favorece la experiencia, calidad y know-how } \\
\text { de los empleados. }\end{array}$ & $\begin{array}{l}\text { España obtiene una capacidad instalada de energía } \\
\text { eólica, en el año } 2010 \text {, de } 20.759 \text { MW. Esto per- } \\
\text { mite un abastecimiento de la demanda del } 15,3 \text { por } \\
100 \text { en tal país (CNE, 2011; Eurobserver, 2011). } \\
\text { De nuevo, la legislación ha sido un elemento cla- } \\
\text { ve en el desarrollo de este tipo de tecnología de } \\
\text { producción en España. Destacan las siguientes } \\
\text { regulaciones: } \\
\text { Real Decreto 2.818/1998, Real Decreto 436/2004, } \\
\text { Real Decreto 661/2007. Permiten la expansión eó- } \\
\text { lica mediante el establecimiento de dos opciones a } \\
\text { los generadores de esta tecnología de producción-la } \\
\text { venta de su electricidad a una tarifa regulada o su } \\
\text { entrada en el mercado de la que obtendría una pri- } \\
\text { ma junto con el precio del mercado (estableciéndose } \\
\text { unos umbrales de rentabilidad mínimos y máximos). } \\
\text { Real Decreto 1.614/2010. Establece una limita- } \\
\text { ción de las horas equivalentes de funcionamiento } \\
\text { de las plantas eólicas con derecho a prima (2.589 } \\
\text { horas/año para las instalaciones de energía eólica } \\
\text { en tierra). Asimismo, conlleva una reducción de la } \\
\text { prima de referencia del } 35 \text { por } 100 \text { para las instala- } \\
\text { ciones de energía eólica acogidas al Real Decreto } \\
661 / 2007 \text { y aquellas de potencia superior a } 50 \text { MW. } \\
\text { No obstante, el Real Decreto-ley } 1 / 2012 \text { conlleva } \\
\text { una suspensión de los procedimientos de prea- } \\
\text { signación de retribución y la supresión de los } \\
\text { incentivos económicos para nuevas instalaciones } \\
\text { de producción de energía eólica. } \\
\text { Las anteriores normativas han supuesto la crea- } \\
\text { ción de compañías competitivas que están ac- } \\
\text { tualmente activas en los mercados de tecnología } \\
\text { internacional. }\end{array}$ \\
\hline
\end{tabular}

NOTA: * La repotenciación (repowering) hace referencia a la sustitución de la primera generación de turbinas eólicas por turbinas modernas de multimegavatios, lo que permitiría duplicar la capacidad en tierra firme con una menor cantidad de turbinas.

FUENTE: Elaboración propia. 


\section{CUADRO 8}

\section{COMPARACIÓN DEL SISTEMA DE PRIMAS DE LA ENERGÍA SOLAR FOTOVOLTAICA EN ALEMANIA Y ESPAÑA}

\begin{tabular}{|c|c|}
\hline Alemania & España \\
\hline $\begin{array}{l}\text { Alemania tiene una capacidad instalada de ener- } \\
\text { gía solar fotovoltaica, en el año } 2010 \text {, de } 17.370 \\
\text { MW. Esto permite un abastecimiento de la de- } \\
\text { manda del } 2 \text { por } 100 \text { en dicho Estado Miembro } \\
\text { (Eurobserver, 2011). } \\
\text { La explicación a este importante desarrollo de } \\
\text { energía solar fotovoltaica en Alemania viene } \\
\text { dado de forma relevante por la legislación apli- } \\
\text { cada para la promoción de tal tecnología de pro- } \\
\text { ducción. Destaca la siguiente normativa: } \\
\text { Sistema de apoyo a la tarifa (1991). Los resul- } \\
\text { tados obtenidos no son tan satisfactorios, como } \\
\text { en el caso de la energía eólica, debido a que el } \\
\text { importe de la prima (valor inicial: } 8,5 \text { céntimos } \\
\text { euro/kWh) no estaba cercano a los costes de esta } \\
\text { tecnología de producción. } \\
\text { Programa de } 1.000 \text { Tejados Fotovoltaicos (1991- } \\
\text { 1995). Debido a la utilización de recursos, alter- } \\
\text { nativos a los derivados del marco regulatorio, } \\
\text { tales como los desarrollados por los activistas } \\
\text { solares y las empresas municipales, se consiguió } \\
\text { el objetivo de este programa. } \\
\text { Programa de 100.000 Tejados Fotovoltaicos } \\
\text { (1999). } \\
\text { Ley de Energías Renovables (2000) (y sus en- } \\
\text { miendas en los años } 2004 \text { y 2009). Conllevan un } \\
\text { incremento de la prima para compensar la pér- } \\
\text { dida de incentivos del Programa de los } 100.000 \\
\text { Tejados Fotovoltaicos, situándola con un impor- } \\
\text { te de } 57 \text { céntimos euro/kWh. Esto conlleva un } \\
\text { boom en la inversión de este tipo de renovables } \\
\text { lo que hace que la segunda enmienda de la Ley } \\
\text { de Energías Renovables (2009) conlleve un des- } \\
\text { censo de la tarifa entre el } 8 \text { por } 100 \text { y el } 10 \text { por } \\
100 \text { en el año } 2010 \text { y, a partir de dicho año, un } 10 \\
\text { por } 100 \text { anualmente. } \\
\text { Esta legislación ha supuesto un importante desa- } \\
\text { rrollo de pequeñas instalaciones debido a que la } \\
\text { primera enmienda de la Ley de Energías Renova- } \\
\text { bles (2004) ofrece mayores primas a las peque- } \\
\text { ñas instalaciones. }\end{array}$ & $\begin{array}{l}\text { España obtiene una capacidad instalada de ener- } \\
\text { gía solar fotovoltaica, en el año } 2010 \text {, de } 3.916 \\
\text { MW. Esto permite un abastecimiento de la de- } \\
\text { manda del } 2,4 \text { por } 100 \text { en tal país (CNE, 2011; } \\
\text { Eurobserver, 2011). } \\
\text { De nuevo, la legislación ha sido un elemento cla- } \\
\text { ve en el desarrollo de este tipo de tecnología de } \\
\text { producción en España. Destacan las siguientes } \\
\text { regulaciones: } \\
\text { Real Decreto } 2.818 / 1998, \text { Real Decreto } \\
\text { 436/2004, Real Decreto 661/2007. Esta regu- } \\
\text { lación fija un sistema de incentivos basado en } \\
\text { primas para la promoción de la energía solar fo- } \\
\text { tovoltaica. } \\
\text { Real Decreto 1.578/2008. Establece un nuevo } \\
\text { sistema de primas para esta tecnología de produc- } \\
\text { ción donde su importe viene dado en función de } \\
\text { su ubicación: en cubierta o en suelo. A su vez, las } \\
\text { instalaciones son clasificadas en tipo I y tipo II en } \\
\text { función de su posición, material de construcción } \\
\text { y uso. Esto permite promover la integración de } \\
\text { sistemas fotovoltaicos más pequeños (en cubier- } \\
\text { ta) a través del establecimiento de una prima más } \\
\text { elevada (34 céntimos euro/kWh con respecto a } \\
32 \text { céntimos euro/kWh para las instalaciones de } \\
\text { mayor tamaño). En el caso de las instalaciones en } \\
\text { suelo, se establece una única tarifa con la finali- } \\
\text { dad de evitar problemas relacionados con el dife- } \\
\text { rencial de energía y estimular aquellos proyectos } \\
\text { que maximizan su eficiencia desde una perspecti- } \\
\text { va tecnológica y económica (Mir, 2012). Una vez } \\
\text { que los objetivos sean alcanzados, las primas se } \\
\text { reducirán cada tres meses. } \\
\text { Real Decreto } 14 / 2010 \text { limita el número de horas } \\
\text { de funcionamiento de las instalaciones fotovol- } \\
\text { taicas con derecho a tarifa. } \\
\text { Real Decreto } 1.003 / 2010 \text { crea una categoría es- } \\
\text { pecífica referente a las instalaciones que no cum- } \\
\text { plieron los requisitos establecidos en las anterio- } \\
\text { res legislaciones. } \\
\text { Real Decreto-ley } 1 / 2012 \text { conlleva una suspensión } \\
\text { de los procedimientos de preasignación de retri- } \\
\text { bución y la supresión de los incentivos económi- } \\
\text { cos para nuevas instalaciones de producción de } \\
\text { energía solar fotovoltaica. }\end{array}$ \\
\hline
\end{tabular}

FUENTE: Elaboración propia. 


\section{CUADRO 9 \\ COMPARACIÓN DEL SISTEMA DE PRIMAS DE LA ENERGÍA SOLAR TÉRMICA EN ALEMANIA Y ESPAÑA}

\begin{tabular}{|l|}
\hline \multicolumn{1}{|c|}{ Alemania } \\
\hline Alemania tiene una capacidad instalada de ener- \\
gía solar térmica, en el año 2010, de 9.831 MW. \\
Esto permite un abastecimiento de la demanda \\
del 0,1 por 100 en dicho Estado Miembro (Euro- \\
bserver, 2011). \\
La explicación a este importante desarrollo de \\
energía solar térmica en Alemania viene dado de \\
forma relevante por la legislación aplicada para \\
la promoción de tal tecnología de producción. \\
Destaca la siguiente normativa: \\
Programa de incentivos para el mercado (1999). \\
Busca fomentar la generación de calor solar para \\
calentar agua y para la calefacción de uso domés- \\
tico. Para ello, la Oficina Federal de Economía \\
y Control de Exportaciones apoyaba, a través de \\
las subvenciones, a pymes e inversores privados \\
para que invirtieran en este tipo de tecnología. \\
No obstante, el recorte de subvenciones hizo que \\
se convirtiera en un proceso frecuentemente in- \\
terrumpido. \\
Ley de Energía Renovable Térmica (2008). Otor- \\
ga seguridad en las inversiones de esta tecnología \\
de producción mediante el establecimiento de un \\
presupuesto constante de hasta 500 millones de \\
euros anuales (2009-2012). Asimismo, esta ley \\
introduce obligaciones para los nuevos edificios \\
de cubrir un porcentaje mínimo del consumo de \\
calefacción a partir de energía renovable.
\end{tabular}

Alemania tiene una capacidad instalada de energía solar térmica, en el año 2010, de 9.831 MW. Esto permite un abastecimiento de la demanda del 0,1 por 100 en dicho Estado Miembro (Eurobserver, 2011).

La explicación a este importante desarrollo de energía solar térmica en Alemania viene dado de forma relevante por la legislación aplicada para la promoción de tal tecnología de producción. Destaca la siguiente normativa:

Busca fomentar la generación de calor solar para calentar agua y para la calefacción de uso domésy Control de Exportaciones apoyaba, a través de las subvenciones, a pymes e inversores privados para que invirtieran en este tipo de tecnología. se convirtiera en un proceso frecuentemente interrumpido.

ga seguridad en las inversiones de esta tecnología de producción mediante el establecimiento de un euros anuales (2009-2012). Asimismo, esta ley introduce obligaciones para los nuevos edificios calefacción a partir de energía renovable.
España obtiene una capacidad instalada de energía solar térmica, en el año 2010, de 1.543 MW. Esto permite un abastecimiento de la demanda del 0,4 por 100 en tal país (CNE, 2011; Eurobserver, 2011).

De nuevo, la legislación ha sido un elemento clave en el desarrollo de este tipo de tecnología de producción en España. Destacan las siguientes regulaciones:

Real Decreto 2.818/1998, Real Decreto 436/2004, Real Decreto 661/2007. Esta regulación fija un sistema de incentivos basado en primas para la promoción de esta tecnología de producción.

Nuevo Código Técnico de la Edificación (CTE) (2006). Esta nueva regulación convierte a España en uno de los países con legislación solar más avanzada (EREC, 2008). La importancia de esta legislación viene dada por el éxito de previas ordenanzas municipales solares. El CTE incluye las siguientes áreas principales: seguridad de la estructura de construcción, cuestiones de seguridad y salud, sostenibilidad y eficiencia energética de la construcción. La última parte (Documento Básico Ahorro de Energía) va más allá del nivel mínimo de implantación de la Directiva Comunitaria Europea sobre la gestión de energía de construcciones e incluye la obligación de cubrir el 30-70 por 100 de la demanda doméstica de agua caliente con energía térmica solar.

Plan de Fomento de Energías Renovables 2005 2010. Este plan conlleva, a partir del año 2008, el desarrollo de incentivos financieros para la promoción de estas plantas solares térmicas (la subvención cubre el 37 por 100 de los costes totales del proyecto).

Real Decreto 1614/2010. Establece una limitación de las horas equivalentes de funcionamiento de las plantas solares térmicas con derecho a prima que variará en función de la tecnología que utilicen.

Real Decreto-ley 1/2012. Conlleva una suspensión de los procedimientos de preasignación de retribución y la supresión de los incentivos económicos para nuevas instalaciones de producción de energía solar térmica.

FUENTE: Elaboración propia. 
A partir de las características del marco regulatorio de Alemania comentadas en los Cuadros 7, 8 y 9 se muestran los principales resultados derivados de su aplicación. Así, su uso ha permitido pasar de una cuota de las energías renovables en el consumo de electricidad del 6 por 100 en el año 2000 a un valor del 15 por 100 en el año 2008 (por tanto, el objetivo establecido por el Gobierno Federal de obtener, al menos un 12,5 por 100 en el año 2010, recogido también en la Directiva 2001/77/ EC, se ha superado con creces en el año 2007) (Büsgen y Dürrschmidt, 2008). En el año 2011, las energías renovables en Alemania suponen el 12,2 por 100 del consumo de energía final total y el 20 por 100 del consumo de electricidad (PBL Netherlands Environmental Assessment Agency, 2012).

También se ha observado un incremento en el empleo dentro de la industria de energía renovable. La creación de empleo en este sector asciende en Alemania a 280.000 puestos de trabajo en el año 2008. Aproximadamente un 60 por 100 de estos trabajos fueron creados como resultado de la Ley de Energías Renovables (donde la mayoría se correspondieron con el sector eólico) (BMU, 2008). En el año 2010, Alemania obtiene unas cifras de empleo de 96.100 puestos de trabajo en energía eólica, 13.100 puestos de trabajo en energía solar térmica y 107.800 puestos de trabajo en energía solar fotovoltaica donde se incluyen tanto empleos directos como inducidos (Eurobserver, 2011). Además, Alemania se caracteriza por tener una elevada cualificación de la mano de obra lo que viene dado, en buena medida, por la implantación de un sistema educativo universitario donde se ofertan más de 240 titulaciones centradas en las energías renovables.

El marco regulatorio descrito previamente ha sido un elemento primordial en la protección climática de Alemania. Así, las emisiones de dióxido de carbono $\left(\mathrm{CO}_{2}\right)$ son evitadas en 1,7 billones de toneladas en el año 2011 (donde se incluye la hidráulica) (PBL Netherlands Environmental Assessment Agency, 2012). Asimismo, se han observado reducciones de costes y mejoras de calidad. La expansión de la producción de sistemas de energía eólicos e instalaciones fotovoltaicas en Alemania ha permitido una mayor oportunidad de explotar reducciones de costes. Igualmente, el desarrollo tecnológico - parcialmente promovido por los fondos de investigación suministrados por el Gobierno- ha conducido al desarrollo de tecnologías de energía renovable más eficientes y fiables.

En el caso de España, las características del marco regulatorio, establecidas en los Cuadros 7, 8 y 9, muestran también una serie de aspectos positivos para la economía española. En este contexto, se observa una contribución positiva de las energías renovables al PIB en España. Este sector se sitúa como uno de los motores de la economía española, alcanzando el 0,94 por 100 del PIB nacional en el año 2010, con una contribución cercana a los 10.000 millones de euros. De este importe, el 67 por 100 viene dado por una contribución directa de las energías renovables al PIB y el 23 por 100 restante representa el efecto arrastre que conlleva a otras áreas (como producción de maquinaria o fabricación de componentes eléctricos) (Deloitte-APPA, 2010).

Asimismo, se ha producido un importante desarrollo de la actividad de I+D+i. El elevado crecimiento de algunas tecnologías de producción renovable ha conllevado 
la creación de potentes sectores industriales de fabricación de equipos y componentes con un perfil innovador.

El marco regulatorio previamente descrito ha sido un elemento primordial en la protección climática de España. Así, en el periodo 2005-2010, se evitó la emisión de 145 millones de toneladas de $\mathrm{CO}_{2}$ y un ahorro de 2.483 millones de euros (DeloitteAPPA, 2010). Asimismo, se produce un incremento en el empleo ${ }^{4}$ dentro de la industria de energía renovable. En el año 2010, España tiene unas cifras de empleo en energía eólica de 30.750 trabajadores, en energía solar térmica de 6.000 y en solar fotovoltaica de 19.500, donde se incluyen tanto empleos directos como inducidos (Eurobserver, 2011).

\section{Conclusiones}

En los últimos años, estamos asistiendo a un proceso de desarrollo de las energías renovables en la Unión Europea con la finalidad de aprovechar sus ventajas. Entre las mismas, se encuentran las bajas emisiones de carbono de este tipo de energía y su sostenibilidad con respecto a la procedente de fuentes fósiles. Con la finalidad de aprovechar tales ventajas, es necesaria la participación del gobierno en la fase inicial de las energías renovables para asegurar su desarrollo y protegerlas de la competencia directa de las tecnologías convencionales. Para ello se han fijado tres tipos de sistemas de apoyo a las mismas -el establecimiento de un sistema de primas, el desarrollo de un sistema de pujas competitivas y el establecimiento de certificados verdes-, dando libertad las directivas europeas a los Estados Miembros para su elección.

En el presente trabajo, se analizan las principales características y los resultados obtenidos con la regulación referente a la promoción de energía eólica y energía solar fotovoltaica y térmica en los casos de Alemania y España. La selección de estos países, para realizar el estudio, se debe a que son los primeros países europeos en potencia instalada y producción en tales tecnologías de producción.

Ambos países se caracterizan por utilizar un sistema de primas para la promoción de energías renovables. Este mecanismo permite que los productores de estas tecnologías de producción obtengan, junto con el precio del mercado de electricidad, un incentivo que compensa el valor ambiental que conlleva. Su éxito puede apreciarse, por ejemplo, en el caso de la energía eólica de los países analizados, que se caracterizan por haber presentado el mayor crecimiento europeo en esta tecnología de producción.

Los resultados obtenidos en tales países ponen de manifiesto un importante impulso económico-social. Así, en ambos casos, el impulso de las energías renovables conllevan: $a$ ) un elemento de protección medioambiental (mediante una importante

4 No obstante, la elevada incertidumbre regulatoria referente a los mecanismos de promoción de energía renovable así como los cambios legislativos (algunos de los cuales tienen carácter retroactivo) han conllevado problemas con la financiación de proyectos. Esto ha supuesto una pérdida de 20.000 empleos en el período 2008-2010 (Deloitte-APPA, 2010). 
reducción de emisiones contaminantes), $b$ ) un considerable incremento de empleo, c) una contribución positiva al PIB y d) el desarrollo de la actividad de I+D+i con la consiguiente creación de un potente sector industrial de fabricación y componentes. El éxito del mecanismo de primas viene reflejado también en el hecho que supone que más de cuarenta países hayan copiado tal modelo.

Por tanto, podemos concluir indicando que el establecimiento de este sistema de promoción de energía renovable permite el desarrollo de tecnologías de producción renovable (especialmente la energía eólica y la energía solar fotovoltaica). No obstante, a largo plazo, puede convertirse en un problema debido a la dificultad para su financiación (especialmente relevante cuando el porcentaje de energía verde es elevado sobre el total de la energía producida).

De ahí, que una vez que el proceso de recursos renovables esté en una etapa de madurez, algunos estudios muestran que el mejor mecanismo para promover el uso de estas tecnologías de producción es internalizar los costes asociados al uso de los recursos no renovables (bien mediante el uso de tasas o impuestos o bien mediante la introducción de un sistema armonizado de certificados verdes negociables donde se incluyan los beneficios ambientales de los recursos renovables frente a los no renovables (Ciarreta-Antuñano y Gutiérrez-Hita, 2009).

\section{Referencias bibliográficas}

[1] BMU (Bundesministerium für Umweltz, Naturschutz and Reaktorsicherheit) (2008): Renewable Energy Sources in Figures. National and International Development (disponible en www.erneuerbare-energien.de, www.feed-in-cooperation.org).

[2] BÜSGEN, U. y DÜRRSCHMIDT, W. (2008): «The expansion of electricity generation from renewable energies in Germany. A review based on the renewable energy sources act report 2007 and the new German feed-in legislation», Energy Policy, vol. 37, pp. 2536-2545.

[3] CASTRO-RODRÍGUEZ, F. y SIOTIS, G. (2010): «El efecto del poder de mercado sobre la inversión en generación en mercados eléctricos liberalizados», Cuadernos Económicos de ICE, n. ${ }^{\circ}$ 79, pp. 139-159.

[4] CIARRETA-ANTUÑANO, A. y GUTIÉRREZ-HITA, C. (2008): «Recursos renovables en el mercado eléctrico español: instrumentos y efectos», Cuadernos Económicos de ICE, n. ${ }^{\circ} 79$, pp. 161-185.

[5] CNE (2011): La energía en España en 2010. Ministerio de Turismo, Industria y Comercio. Madrid.

[6] DEL RÍO, P. (2008): «Ten years of renewable electricity policies in Spain: an analysis of successive feed-in tariff reforms», Energy Policy, vol. 36, pp. 2917-2929.

[7] DELOITTE-APPA (2010): Estudio macroeconómico del impacto de las energías renovables en la economía española. Asociación de Productores de Energía Renovable. Madrid.

[8] DOSI, G. (1988): «The nature of the innovative process», en Dosi et al.(eds.), Technical Change and Economic Theory. Pinter: London. 
[9] EREC (EUROPEAN RENEWABLE ENERGY COUNCIL) (2008): Renewable Energy Policy Review: Spain. European Renewable Energy Council. Bruselas.

[10] EUROBSERVER (2011): The State of Renewable Energies in Europe. 11 th Eurobserver Report. Francia.

[11] FAGES, J. y BUSTOS, M. (2003): Introducción a los sistemas de retribución de las renovables en la Unión Europea. La visión de los productores de energía renovable. APPA. Barcelona.

[12] LAUBER, V. y MEZ, L. (2004): «Three decades of renewable electricity policies in Germany», Energy\&Environment, vol. 15, n. . 4, pp. 599-623.

[13] MENANTEAU, P.; FINON, D. y LAMI, M. L. (2003): «Prices versus quantities: choosing policies for promoting the development of renewable energy», Energy Policy, vol. 31, pp. 799-812.

[14] MIR, P. (2012): «La regulación fotovoltaica y solar termoeléctrica en España», Cuadernos Económicos de ICE, n. ${ }^{\circ}$ 83, pp. 185-205.

[15] MORENO, B. y LÓPEZ, A. J. (2008): «The effects of renewable energy on employment. The case of Asturias (Spain)», Renewable \& Sustainable Energy Reviews, vol. 12, pp. 732-751.

[16] PBL NETHERLANDS ENVIRONMENTAL ASSESSMENT AGENCY (2012): Trends in Global $\mathrm{Co}_{2}$ Emissions 2012 Report. PBL Netherlands Environmental Assessment Agency. The Hague.

[17] VARELA, L. y GARCÍA ÁLVAREZ, M. T (2010): «La reforma fiscal verde en España: un nuevo impulso fiscal», Boletín ICE Económico, n. ${ }^{\circ}$ 3.002, pp. 27-38.

[18] VERA, B. (2012): «Economía de las energías eólicas», Cuadernos Económicos de ICE, n. ${ }^{\circ} 83$, pp. $165-182$. 



\section{SUSCRIPCIÓN ANUAL}

CUADERNOS ECONÓMICOS DE INFORMACIÓN COMERCIAL (2 NÚMEROS)

\begin{tabular}{|c|c|c|c|}
\hline & $\begin{array}{c}\text { ESPAÑA } \\
\mathbf{1} \text { año }\end{array}$ & $\begin{array}{c}\text { UNIÓN EUROPEA } \\
\mathbf{1} \text { año }\end{array}$ & $\begin{array}{c}\text { RESTO DEL } \\
\text { MUNDO } \\
\mathbf{1} \text { año }\end{array}$ \\
\hline SUSCRIPCIÓN & $30,00 €$ & $40,00 €$ & $40,00 €$ \\
\hline $\begin{array}{c}\text { Gastos de envío } \\
\text { España }\end{array}$ & $2,36 €$ & $10,04 €$ & $16,10 €$ \\
\hline $\begin{array}{c}\text { Más 4\% de IVA. } \\
\text { Excepto Canarias, Ceuta y Melilla }\end{array}$ & $1,29 €$ & & \\
\hline TOTAL & $\mathbf{3 3 , 6 5} €$ & $\mathbf{5 0 , 0 4} €$ & $\mathbf{5 6 , 1 0} €$ \\
\hline & & & \\
\hline
\end{tabular}

\section{EJEMPLARES SUELTOS}

\begin{tabular}{|c|c|c|c|}
\hline \multicolumn{4}{|c|}{ CUADERNOS ECONÓMICOS DE INFORMACIÓN COMERCIAL } \\
\hline & $\begin{array}{c}\text { ESPAÑA } \\
1 \text { ejemplar }\end{array}$ & $\begin{array}{c}\text { UNIÓN EUROPEA } \\
1 \text { ejemplar }\end{array}$ & $\begin{array}{c}\text { RESTO DEL } \\
\text { MUNDO } \\
\text { ejemplar }\end{array}$ \\
\hline NÚMERO SUELTO & $18,00 €$ & $23,00 €$ & $23,00 €$ \\
\hline $\begin{array}{c}\text { Gastos de envío } \\
\text { España }\end{array}$ & $1,18 €$ & $5,02 €$ & $8,05 €$ \\
\hline $\begin{array}{c}\text { Más 4\% de IVA. } \\
\text { Excepto Canarias, Ceuta y Melilla }\end{array}$ & $0,77 €$ & $\mathbf{2 8 , 0 2} €$ & $31,05 €$ \\
\hline TOTAL & $\mathbf{1 9 , 9 5 €}$ & & \\
\hline
\end{tabular}

\section{DATOS}

Nombre y apellidos

Empresa

Domicilio

D.P.

N.I.F.

Teléf.

Email
Transferencia a la cuenta de ingresos por venta de publicaciones del Ministerio de Economía y Competitividad.

IBERCAJA. Calle Alcalá 29. MADRID (ESPAÑA) CÓDIGO CUENTA CLIENTE: 2085-9252-07-0330598330

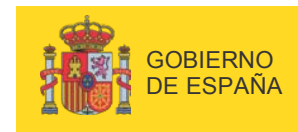

\section{MINISTERIO \\ DE ECONOMIA Y \\ COMPETITIVIDAD}

Suscripciones y ventas por correspondencia:

Paseo de la Castellana, 162, $8^{\text {a }}$ Planta 28046 Madrid. Teléfono 91583.55.07

Suscripciones a través de la página web del Ministerio de Economía y Competitividad DistribucionPublicaciones@mineco.es 